\title{
OBSERVATION OF LOW-DEGREE MODES FROM SOHO/MDI USING OPTIMAL MASKS
}

\author{
T. TOUTAIN \\ Nice Observatory \\ B.P. 4229, F-06304 Nice cedex \\ A. KOSOVICHEV \\ HEPL \\ Stanford University, USA \\ AND \\ T. APPOLRCHAUX \\ ESTEC, ESA, The Netherlands
}

The overlap of peaks of split multiplets in oscillation power spectra and the leakage of other degree modes could significantly affect the measurements of the rotational frequency splitting. We have developed optimal masks which allow to isolate individual components of the multiplets. The method is applied to the Michelson Doppler Imager Low-l data. The results of the mode frequency measurements are reported.

\section{Optimal masks}

The MDI instrument provides, among others, a LOI-proxy velocity signal (Scherrer et al.(1995)) consisting of 180 mean velocities, one for each bin of the CCD detector. The signal gathered over 1 year (May 96 to April 97) has a duty cycle better than $99 \%$. Because we are interested only in very low degree modes $(l \leq 3)$ we reduce the time series by binning up the 180 pixels to get to the 12 scientific pixels of the LOI (Appourchaux et al., 1997). Instead of applying spherical harmonic masks to the time series we follow Kosovichev (1986) and apply a Singular Value Decomposition technique (equivalent to using the inverse of the leakage matrix) to get optimal masks. Figure 1 shows a $l=1 m-\nu$ diagram obtained using optimal masks. Crosstalks between modes have disappeared making the fitting of lines easier. Modes are fitted in the Fourier domain, as described by Appourchaux and Gizon(1997). Table I gives the central frequencies we obtain.

\section{References:}

Appourchaux T. et al., 1997, Sol. Phys. 170, 24

Appourchaux, T. and Gizon, L., 1997, these proceedings

Kosovichev, A.G. 1986, Bull. Crimean Astrophys. Obs.75, 19

Scherrer,P.H.,et al., 1995, Sol. Phys. 162, 129 


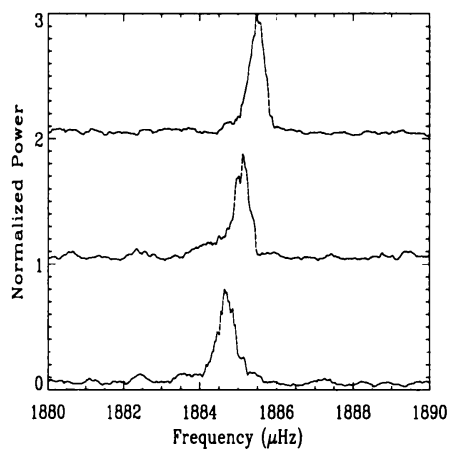

Figure 1. Smoothed $m-\nu$ diagram for $l=1$ using optimal masks on the 1-year MDI time series

TABLE 1. Central frequencies (in $\mu \mathrm{Hz}$ ) with their 1- $\sigma$ error bars

\begin{tabular}{ccccc}
\hline order & $l=0$ & $l=1$ & $l=2$ & $l=3$ \\
\hline 10 & $1548.31 \pm 0.07$ & & $1674.51 \pm 0.20$ & $1729.06 \pm 0.03$ \\
11 & $1686.58 \pm 0.04$ & $1749.26 \pm 0.06$ & $1810.27 \pm 0.04$ & $1865.28 \pm 0.03$ \\
12 & $1822.20 \pm 0.05$ & $1885.06 \pm 0.03$ & $1945.76 \pm 0.03$ & $2001.19 \pm 0.04$ \\
13 & $1957.40 \pm 0.05$ & $2020.76 \pm 0.03$ & $2082.08 \pm 0.03$ & $2137.75 \pm 0.03$ \\
14 & $2093.46 \pm 0.05$ & $2156.78 \pm 0.04$ & $2217.60 \pm 0.03$ & $2273.34 \pm 0.03$ \\
15 & $2228.67 \pm 0.06$ & $2291.92 \pm 0.04$ & $2352.22 \pm 0.03$ & $2407.56 \pm 0.03$ \\
16 & $2362.74 \pm 0.06$ & $2425.46 \pm 0.03$ & $2485.80 \pm 0.03$ & $2541.53 \pm 0.03$ \\
17 & $2496.06 \pm 0.06$ & $2559.06 \pm 0.04$ & $2619.51 \pm 0.03$ & $2676.13 \pm 0.03$ \\
18 & $2629.81 \pm 0.06$ & $2693.26 \pm 0.03$ & $2754.46 \pm 0.03$ & $2811.47 \pm 0.03$ \\
19 & $2764.10 \pm 0.06$ & $2828.06 \pm 0.04$ & $2889.58 \pm 0.03$ & $2947.00 \pm 0.03$ \\
20 & $2898.91 \pm 0.03$ & $2963.24 \pm 0.03$ & $3024.70 \pm 0.03$ & $3082.19 \pm 0.03$ \\
21 & $3033.73 \pm 0.07$ & $3098.04 \pm 0.04$ & $3159.76 \pm 0.03$ & $3217.66 \pm 0.03$ \\
22 & $3168.57 \pm 0.06$ & $3233.02 \pm 0.04$ & $3294.93 \pm 0.04$ & $3353.41 \pm 0.04$ \\
23 & $3303.36 \pm 0.07$ & $3368.64 \pm 0.05$ & $3430.66 \pm 0.05$ & $3489.44 \pm 0.06$ \\
24 & $3438.88 \pm 0.08$ & $3504.06 \pm 0.07$ & $3566.73 \pm 0.07$ & $3625.93 \pm 0.07$ \\
25 & $3574.40 \pm 0.18$ & $3640.25 \pm 0.10$ & $3702.90 \pm 0.12$ & $3762.67 \pm 0.10$ \\
26 & $3710.36 \pm 0.22$ & $3776.31 \pm 0.20$ & $3839.57 \pm 0.20$ & $3899.53 \pm 0.15$ \\
27 & $3846.96 \pm 0.33$ & $3912.85 \pm 0.20$ & $3976.31 \pm 0.28$ & $4174.71 \pm 0.35$ \\
\hline
\end{tabular}

